(C) 2005 American Chemical Society, Organometallics, Daiss om040143k Supporting Info Page 1

\title{
$0 \mathrm{MO} 040143 \mathrm{~K}$
}

\section{Supporting Information}

\section{Synthesis, Crystal Structure Analysis, and Pharmacological Characterization of Disila-bexarotene, a Disila-Analogue of the RXR-Selective Retinoid Agonist Bexarotene}

\author{
Jürgen O. Daiss, ${ }^{+}$Christian Burschka, ${ }^{+}$John S. Mills, ${ }^{\dagger}$ John G. Montana, ${ }^{\dagger}$ Graham A. Showell, ${ }^{\dagger}$ \\ Ian Fleming, ${ }^{\S}$ Claudine Gaudon, ${ }^{\ddagger}$ Diana Ivanova, ${ }^{\ddagger}$ Hinrich Gronemeyer, ${ }^{\ddagger}$ and Reinhold Tacke ${ }^{*},+$ \\ Institut für Anorganische Chemie, Universität Würzburg, Am Hubland, D-97074 Würzburg, Germany, \\ Paradigm Therapeutics Ltd., 162 Cambridge Science Park, Milton Road, Cambridge CB4 OGP, U.K., \\ Department of Chemistry, Cambridge University, Lensfield Road, Cambridge CB2 IEW, U.K., \\ and Institut de Génétique et de Biologie Moléculaire et Cellulaire (IGMBC), CNRS/INSERM/ULP, \\ BP 10142, F-67404 Illkirch Cedex, C. U. de Strasbourg, France
}

* To whom correspondence should be addressed. Phone: +49-931-888-5250. Fax: +49-931-8884609. E-mail: r.tacke@mail.uni-wuerzburg.de.

${ }^{+}$Institut für Anorganische Chemie, Universität Würzburg.

${ }^{\dagger}$ Paradigm Therapeutics Ltd., Cambridge.

${ }^{\S}$ Department of Chemistry, Cambridge University.

${ }^{\ddagger}$ Department of Cell Biology and Signal Transduction, IGBMC, Illkirch. 
(C) 2005 American Chemical Society, Organometallics, Daiss om040143k Supporting Info Page 2 2

Table 1. Crystal data and structure refinement for $1 a$.

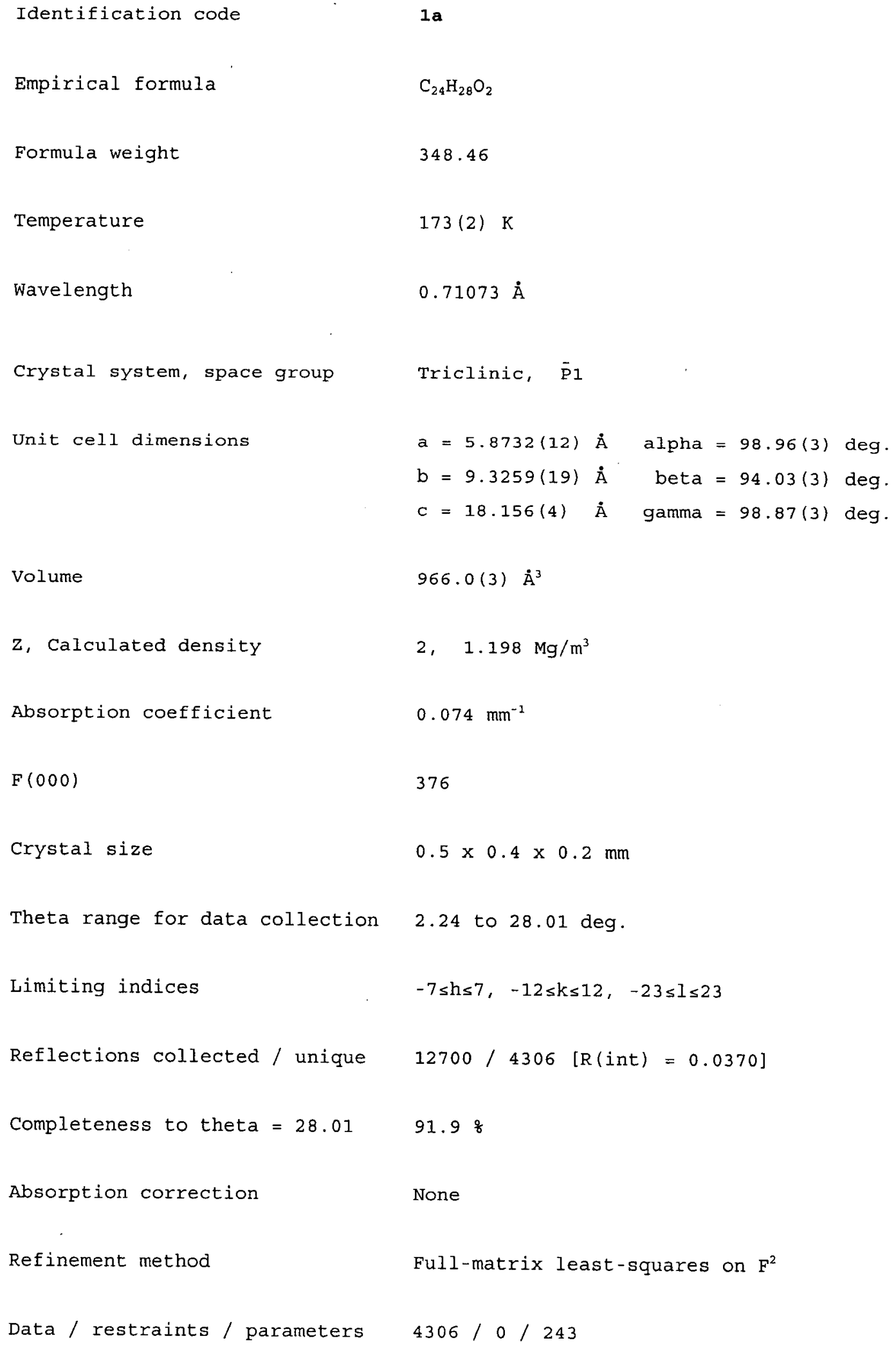


(C) 2005 American Chemical Society, Organometallics, Daiss om040143k Supporting Info Page 3 3

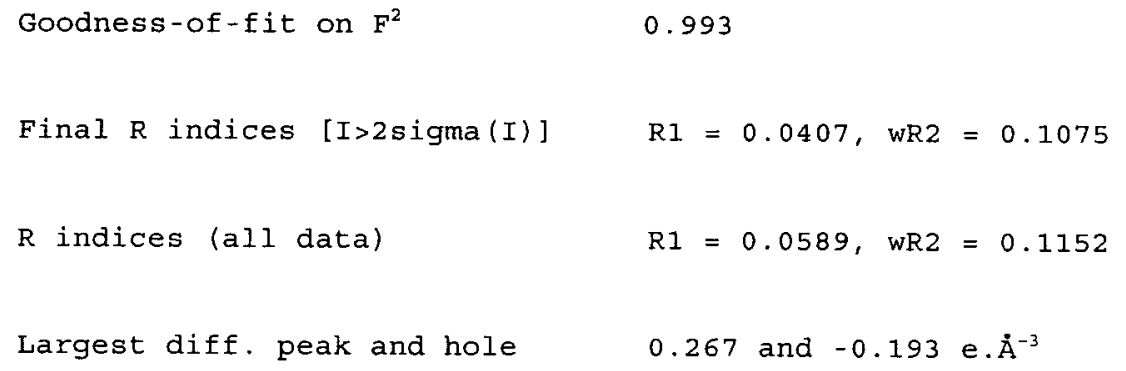


(C) 2005 American Chemical Society, Organometallics, Daiss om040143k Supporting Info Page 4 4

Table 2. Atomic coordinates $\left(x 10^{4}\right)$ and equivalent isotropic displacement parameters $\left(\dot{A}^{2} \times 10^{3}\right)$ for 1a. $U(e q)$ is defined as one third of the trace of the orthogonalized Uij tensor.

\begin{tabular}{|c|c|c|c|c|}
\hline & $x$ & $Y$ & $\mathbf{z}$ & $\mathrm{U}(\mathrm{eq})$ \\
\hline$C(01)$ & $12685(2)$ & $5355(1)$ & $2033(1)$ & $22(1)$ \\
\hline$C(02)$ & $12717(2)$ & $2801(1)$ & $741(1)$ & $23(1)$ \\
\hline$O(I)$ & $11151(2)$ & $-3835(1)$ & $4476(1)$ & $36(1)$ \\
\hline$O(2)$ & $7829(2)$ & $-3802(1)$ & $5007(1)$ & $38(1)$ \\
\hline$C(6)$ & $13868(3)$ & $1454(1)$ & $504(1)$ & $35(1)$ \\
\hline$C(5)$ & $11074(3)$ & $2979(1)$ & $80(1)$ & $32(1)$ \\
\hline$C(4)$ & $14659(2)$ & $4141(1)$ & $957(1)$ & $28(1)$ \\
\hline$c(3)$ & $13730(2)$ & $5506(1)$ & $1289(1)$ & $28(1)$ \\
\hline$C(1)$ & $10948(3)$ & $6422(1)$ & $2142(1)$ & $32(I)$ \\
\hline$C(2)$ & $14618(3)$ & $5783(1)$ & $2679(1)$ & $31(1)$ \\
\hline$C(8)$ & $11425(2)$ & $3777(1)$ & $2022(1)$ & $20(1)$ \\
\hline$C(7)$ & $11352(2)$ & $2631(1)$ & $1417(1)$ & $20(1)$ \\
\hline$C(12)$ & $9932(2)$ & $1287(1)$ & $1433(1)$ & $24(1)$ \\
\hline$C(11)$ & $8695(2)$ & $997(1)$ & $2029(1)$ & $24(1)$ \\
\hline$C(10)$ & $8940(2)$ & $2103(1)$ & $2667(1)$ & $21(1)$ \\
\hline$C(9)$ & $10268(2)$ & $3469(1)$ & $2642(1)$ & $22(1)$ \\
\hline$C(13)$ & $7086(3)$ & $-456(1)$ & $1969(1)$ & $34(1)$ \\
\hline$C(14)$ & $7809(2)$ & $1858(1)$ & $3359(1)$ & $23(1)$ \\
\hline$C(15)$ & $6502(3)$ & $2782(1)$ & $3670(1)$ & $37(1)$ \\
\hline$C(16)$ & $8256(2)$ & $558(1)$ & $3700(1)$ & $21(1)$ \\
\hline$C(17)$ & $10277(2)$ & $-28(1)$ & $3600(1)$ & $24(1)$ \\
\hline$C(18)$ & $10640(2)$ & $-1267(1)$ & $3898(1)$ & $24(1)$ \\
\hline$C(19)$ & $8985(2)$ & $-1941(1)$ & $4303(1)$ & $23(1)$ \\
\hline$C(20)$ & $6974(2)$ & $-1363(1)$ & $4410(1)$ & $25(1)$ \\
\hline$C(21)$ & $6616(2)$ & $-125(1)$ & $4112(1)$ & $24(1)$ \\
\hline$C(22)$ & $9420(2)$ & $-3278(1)$ & $4604(1)$ & $26(1)$ \\
\hline
\end{tabular}


(C) 2005 American Chemical Society, Organometallics, Daiss om040143k Supporting Info Page 5 5

Table 3. Bond lengths [Å] and angles [deg] for $1 \mathrm{a}$.

\begin{tabular}{|c|c|}
\hline$C(01)-C(1)$ & $1.5331(18)$ \\
\hline$C(01)-C(2)$ & $1.5332(19)$ \\
\hline$C(01)-C(8)$ & $1.5380(15)$ \\
\hline$C(01)-C(3)$ & $1.5407(16)$ \\
\hline$C(02)-C(7)$ & $1.5281(16)$ \\
\hline$C(02)-C(5)$ & $1.532(2)$ \\
\hline$C(02)-C(6)$ & $1.5347(18)$ \\
\hline$C(02)-C(4)$ & $1.5367(18)$ \\
\hline$O(1)-C(22)$ & $1.2309(16)$ \\
\hline $\mathrm{O}(2)-\mathrm{H}(2)$ & $0.89(2)$ \\
\hline$O(2)-C(22)$ & $1.3088(16)$ \\
\hline$C(4)-C(3)$ & $1.5170(18)$ \\
\hline$C(8)-C(7)$ & $1.4005(15)$ \\
\hline$C(8)-C(9)$ & $1.4006(16)$ \\
\hline$C(7)-C(12)$ & $1.3996(16)$ \\
\hline$C(12)-C(11)$ & $1.3825(17)$ \\
\hline$C(11)-C(10)$ & $1.4089(16)$ \\
\hline$C(11)-C(13)$ & $1.5114(16)$ \\
\hline$C(10)-C(9)$ & $1.3962(16)$ \\
\hline$C(10)-C(14)$ & $1.4949(16)$ \\
\hline$C(14)-C(15)$ & $1.3278(19)$ \\
\hline$C(14)-C(16)$ & $1.4928(16)$ \\
\hline$C(16)-C(17)$ & $1.3928(18)$ \\
\hline$C(16)-C(21)$ & $1.3974(16)$ \\
\hline$C(17)-C(18)$ & $1.3888(17)$ \\
\hline$C(18)-C(19)$ & $1.3914(17)$ \\
\hline$C(19)-C(20)$ & $1.3854(18)$ \\
\hline$C(19)-C(22)$ & $1.4862(17)$ \\
\hline$C(20)-C(21)$ & $1.3878(17)$ \\
\hline$C(1)-C(01)-C(2)$ & $109.05(10)$ \\
\hline$C(1)-C(01)-C(8)$ & $109.18(10)$ \\
\hline$C(2)-C(01)-C(8)$ & $110.27(10)$ \\
\hline$C(1)-C(01)-C(3)$ & $107.85(10)$ \\
\hline$C(2)-C(01)-C(3)$ & $109.28(11)$ \\
\hline$C(8)-C(01)-C(3)$ & $111.16(9)$ \\
\hline$C(7)-C(02)-C(5)$ & $109.37(11)$ \\
\hline$C(7)-C(02)-C(6)$ & $111.60(10)$ \\
\hline$C(5)-C(02)-C(6)$ & $108.38(11)$ \\
\hline$C(7)-C(02)-C(4)$ & $108.79(9)$ \\
\hline
\end{tabular}


(C) 2005 American Chemical Society, Organometallics, Daiss om040143k Supporting Info Page 6 6

\begin{tabular}{|c|c|}
\hline$C(5)-C(02)-C(4)$ & $111.44(10)$ \\
\hline$C(6)-C(02)-C(4)$ & $107.28(12)$ \\
\hline $\mathrm{H}(2)-\mathrm{O}(2)-\mathrm{C}(22)$ & $109.2(13)$ \\
\hline$C(3)-C(4)-C(02)$ & $111.57(11)$ \\
\hline$C(4)-C(3)-C(01)$ & $112.70(10)$ \\
\hline$C(7)-C(8)-C(9)$ & $118.06(10)$ \\
\hline$C(7)-C(8)-C(01)$ & $123.39(10)$ \\
\hline$C(9)-C(8)-C(01)$ & $118.55(10)$ \\
\hline$C(12)-C(7)-C(8)$ & $118.16(10)$ \\
\hline$C(12)-C(7)-C(02)$ & $119.16(10)$ \\
\hline$C(8)-C(7)-C(02)$ & $122.67(10)$ \\
\hline$C(11)-C(12)-C(7)$ & $123.86(10)$ \\
\hline$C(12)-C(11)-C(10)$ & $117.95(10)$ \\
\hline$C(12)-C(11)-C(13)$ & $119.64(11)$ \\
\hline$C(10)-C(11)-C(13)$ & $122.38(11)$ \\
\hline$C(9)-C(10)-C(11)$ & $118.42(10)$ \\
\hline$C(9)-C(10)-C(14)$ & $119.59(10)$ \\
\hline$C(11)-C(10)-C(14)$ & $121.99(10)$ \\
\hline$C(10)-C(9)-C(8)$ & $123.13(10)$ \\
\hline$C(15)-C(14)-C(16)$ & $121.28(11)$ \\
\hline$C(15)-C(14)-C(10)$ & $121.22(11)$ \\
\hline$C(16)-C(14)-C(10)$ & $117.48(10)$ \\
\hline$C(17)-C(16)-C(21)$ & $118.33(10)$ \\
\hline$C(17)-C(16)-C(14)$ & $121.19(10)$ \\
\hline$C(21)-C(16)-C(14)$ & $120.46(11)$ \\
\hline$C(18)-C(17)-C(16)$ & $120.71(11)$ \\
\hline$C(17)-C(18)-C(19)$ & $120.32(12)$ \\
\hline$C(20)-C(19)-C(18)$ & $119.55(11)$ \\
\hline$C(20)-C(19)-C(22)$ & $121.71(11)$ \\
\hline$C(18)-C(19)-C(22)$ & $118.74(12)$ \\
\hline$C(19)-C(20)-C(2 I)$ & $120.00(11)$ \\
\hline$C(20)-C(21)-C(16)$ & $121.10(12)$ \\
\hline$O(1)-C(22)-O(2)$ & $123.55(11)$ \\
\hline$O(1)-C(22)-C(19)$ & $121.68(11)$ \\
\hline$O(2)-C(22)-C(19)$ & $114.77(12)$ \\
\hline
\end{tabular}


(C) 2005 American Chemical Society, Organometallics, Daiss om040143k Supporting Info Page 7 7

Table 4. Anisotropic displacement parameters $\left(\dot{A}^{2} \times 10^{3}\right)$ for 1a. The anisotropic displacement factor exponent takes the form:

$-2 \pi^{2}\left[h^{2} a *^{2} U 11+\ldots+2 h k a * b * 012\right]$

\begin{tabular}{|c|c|c|c|c|c|c|}
\hline & U11 & $\mathrm{U} 22$ & U33 & U23 & U13 & U12 \\
\hline$C(01)$ & $26(1)$ & $16(1)$ & $25(1)$ & $6(1)$ & $5(1)$ & $1(1)$ \\
\hline$C(02)$ & $28(1)$ & $21(1)$ & $22(1)$ & $5(1)$ & $6(1)$ & $5(1)$ \\
\hline$O(1)$ & $42(1)$ & $33(1)$ & $43(1)$ & $21(1)$ & $15(1)$ & $17(1)$ \\
\hline$O(2)$ & $44(1)$ & $33(1)$ & $49(1)$ & $28(1)$ & $19(1)$ & $15(1)$ \\
\hline$C(6)$ & $40(1)$ & $30(1)$ & $38(1)$ & $5(1)$ & $11(1)$ & $13(1)$ \\
\hline$C(5)$ & $38(1)$ & $34(1)$ & $24(1)$ & $9(1)$ & $2(1)$ & $4(1)$ \\
\hline$C(4)$ & $28(1)$ & $28(1)$ & $28(1)$ & $8(1)$ & $10(1)$ & $1(1)$ \\
\hline$C(3)$ & $35(1)$ & $21(1)$ & $28(1)$ & $9(1)$ & $8(1)$ & $-2(1)$ \\
\hline$C(1)$ & $36(1)$ & $20(1)$ & $42(1)$ & $9(1)$ & $8(1)$ & $5(1)$ \\
\hline$C(2)$ & $33(1)$ & $27(1)$ & $31(1)$ & $8(1)$ & $-1(1)$ & $-6(1)$ \\
\hline$C(8)$ & $21(1)$ & 16 (i) & $22(1)$ & $6(1)$ & $1(1)$ & $2(1)$ \\
\hline$C(7)$ & $23(1)$ & $19(1)$ & $20(1)$ & $7(1)$ & $1(1)$ & $4(1)$ \\
\hline$C(12)$ & $33(1)$ & $18(1)$ & $21(1)$ & $4(1)$ & $1(1)$ & $2(1)$ \\
\hline$C(11)$ & $27(1)$ & $18(1)$ & $25(1)$ & $8(1)$ & $-1(1)$ & $-1(1)$ \\
\hline$C(10)$ & $23(1)$ & $20(1)$ & $22(1)$ & $9(1)$ & $3(1)$ & $4(1)$ \\
\hline$C(9)$ & $26(1)$ & $17(1)$ & $23(1)$ & $5(1)$ & $4(1)$ & $3(1)$ \\
\hline$C(13)$ & $45(1)$ & $23(1)$ & $30(1)$ & $7(1)$ & $1(1)$ & $-10(1)$ \\
\hline$C(14)$ & $25(1)$ & $21(1)$ & $25(1)$ & $10(1)$ & $4(1)$ & $2(1)$ \\
\hline$C(15)$ & $49(1)$ & $31(1)$ & $42(1)$ & $21(1)$ & $22(1)$ & $16(1)$ \\
\hline$C(16)$ & $24(1)$ & $19(1)$ & $20(1)$ & $6(1)$ & $2(1)$ & $1(1)$ \\
\hline$C(17)$ & $23(1)$ & $26(1)$ & $26(1)$ & $11(1)$ & $5(1)$ & $3(1)$ \\
\hline$C(18)$ & $23(1)$ & $25(1)$ & $26(1)$ & $9(1)$ & $3(1)$ & $5(1)$ \\
\hline$C(19)$ & $28(1)$ & $20(1)$ & $21(1)$ & $6(1)$ & $2(1)$ & $4(1)$ \\
\hline$C(20)$ & $30(1)$ & $24(1)$ & $25(1)$ & $11(1)$ & $9(1)$ & $3(1)$ \\
\hline$C(21)$ & $24(1)$ & $24(1)$ & $26(1)$ & $9(1)$ & $7(1)$ & $5(1)$ \\
\hline$C(22)$ & $31(1)$ & $25(1)$ & $24(1)$ & $9(1)$ & $5(1)$ & $6(1)$ \\
\hline
\end{tabular}


(C) 2005 American Chemical Society, Organometallics, Daiss om040143k Supporting Info Page 8 8

Table 5. Hydrogen coordinates $\left(\times 10^{4}\right)$ and isotropic displacement parameters $\left(\dot{A}^{2}\right.$ $\times 10^{3}$ ) for $1 \mathrm{a}$.

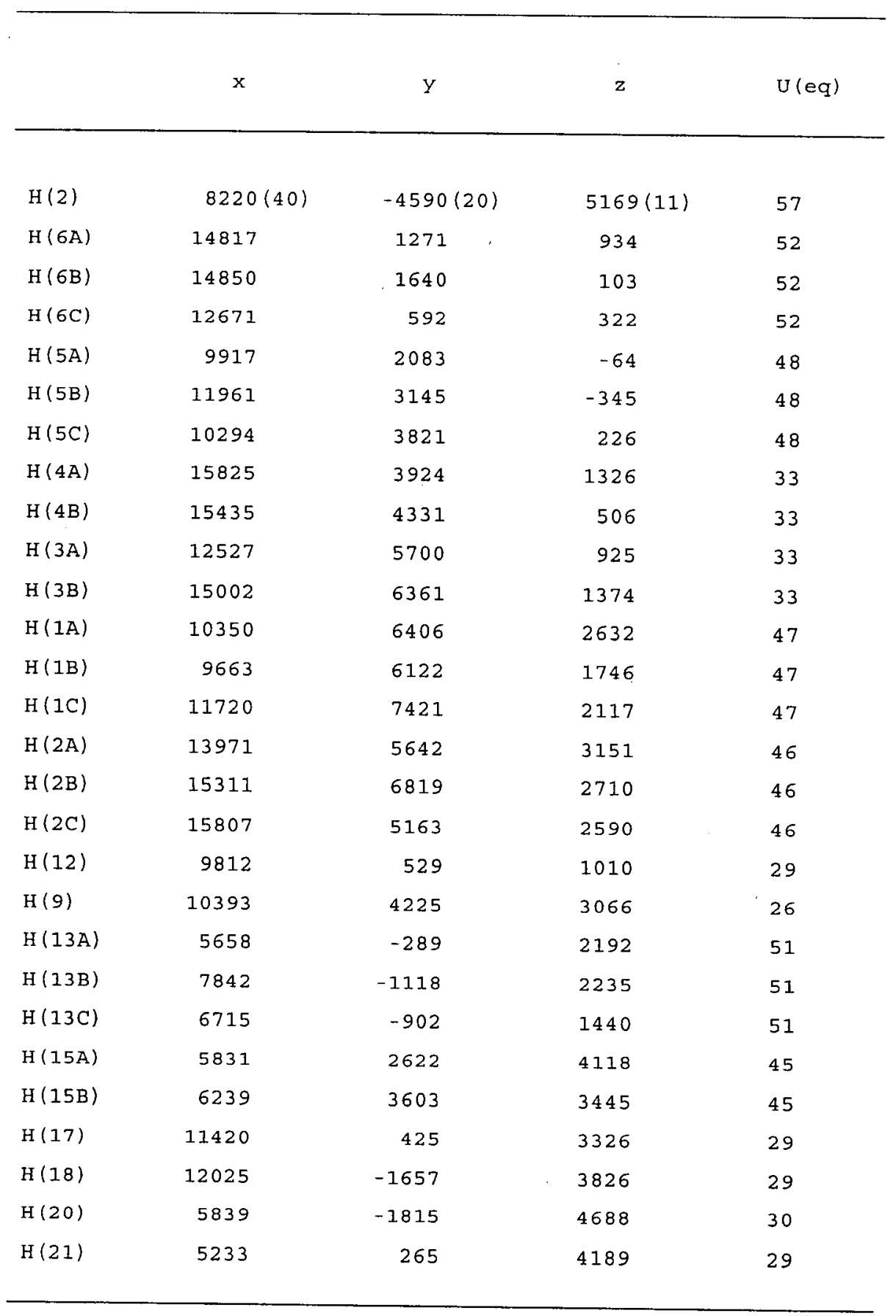


Table 6. Torsion angles [deg] for 1 a.

$$
\begin{aligned}
& C(7)-C(02)-C(4)-C(3) \\
& C(5)-C(02)-C(4)-C(3) \\
& C(6)-C(02)-C(4)-C(3) \\
& \mathrm{C}(02)-\mathrm{C}(4)-\mathrm{C}(3)-\mathrm{C}(01) \\
& C(1)-C(01)-C(3)-C(4) \\
& C(2)-C(01)-C(3)-C(4) \\
& C(8)-C(01)-C(3)-C(4) \\
& C(1)-C(01)-C(8)-C(7) \\
& C(2)-C(01)-C(8)-C(7) \\
& C(3)-C(01)-C(8)-C(7) \\
& C(1)-C(01)-C(8)-C(9) \\
& C(2)-C(01)-C(8)-C(9) \\
& \mathrm{C}(3)-\mathrm{C}(01)-\mathrm{C}(8)-\mathrm{C}(9) \\
& C(9)-C(8)-C(7)-C(12) \\
& \mathrm{C}(01)-\mathrm{C}(8)-\mathrm{C}(7)-\mathrm{C}(12) \\
& C(9)-C(8)-C(7)-C(02) \\
& \mathrm{C}(01)-\mathrm{C}(8)-\mathrm{C}(7)-\mathrm{C}(02) \\
& C(5)-C(02)-C(7)-C(12) \\
& C(6)-C(02)-C(7)-C(12) \\
& \mathrm{C}(4)-\mathrm{C}(02)-\mathrm{C}(7)-\mathrm{C}(12) \\
& \mathrm{C}(5)-\mathrm{C}(02)-\mathrm{C}(7)-\mathrm{C}(8) \\
& C(6)-C(02)-C(7)-C(8) \\
& \mathrm{C}(4)-\mathrm{C}(02)-\mathrm{C}(7)-\mathrm{C}(8) \\
& \mathrm{C}(8)-\mathrm{C}(7)-\mathrm{C}(12)-\mathrm{C}(11) \\
& \mathrm{C}(02)-\mathrm{C}(7)-\mathrm{C}(12)-\mathrm{C}(11) \\
& \mathrm{C}(7)-\mathrm{C}(12)-\mathrm{C}(11)-\mathrm{C}(10) \\
& \mathrm{C}(7)-\mathrm{C}(12)-\mathrm{C}(11)-\mathrm{C}(13) \\
& C(12)-C(11)-C(10)-C(9) \\
& \mathrm{C}(13)-\mathrm{C}(11)-\mathrm{C}(10)-\mathrm{C}(9) \\
& C(12)-C(11)-C(10)-C(14) \\
& C(13)-C(11)-C(10)-C(14) \\
& C(11)-C(10)-C(9)-C(8) \\
& \mathrm{C}(14)-\mathrm{C}(10)-\mathrm{C}(9)-\mathrm{C}(8) \\
& C(7)-C(8)-C(9)-C(10) \\
& \mathrm{C}(01)-\mathrm{C}(8)-\mathrm{C}(9)-\mathrm{C}(10) \\
& \mathrm{C}(9)-\mathrm{C}(10)-\mathrm{C}(14)-\mathrm{C}(15) \\
& \mathrm{C}(11)-\mathrm{C}(10)-\mathrm{C}(14)-\mathrm{C}(15) \\
& \mathrm{C}(9)-\mathrm{C}(10)-\mathrm{C}(14)-\mathrm{C}(16) \\
& C(11)-C(10)-C(14)-C(16) \\
& -52.40(14) \\
& 68.26(13) \\
& -173.26(11) \\
& 64.15 \text { (15) } \\
& -156.19 \text { (11) } \\
& 85.39(13) \\
& -36.55(15) \\
& 121.48(13) \\
& -118.73 \text { (13) } \\
& 2.63(17) \\
& -57.81(15) \\
& 61.98(15) \\
& -176.66(11) \\
& 6.56(18) \\
& -172.73(11) \\
& -174.62(12) \\
& 6.08(19) \\
& 75.57(14) \\
& -44.34(16) \\
& -162.50(12) \\
& -103.24(13) \\
& 136.86(13) \\
& 18.69(16) \\
& -3.7(2) \\
& 177.44(12) \\
& -2.2(2) \\
& 175.88(13) \\
& 4.90(18) \\
& -173.09(12) \\
& -175.59(12) \\
& 6.4(2) \\
& -1.94(19) \\
& 178.53(12) \\
& -3.92(19) \\
& 175.41(12) \\
& 51.66 \text { (19) } \\
& -127.85 \text { (15) } \\
& -126.74 \text { (13) } \\
& 53.75(17)
\end{aligned}
$$


(C) 2005 American Chemical Society, Organometallics, Daiss om040143k Supporting Info Page 10 10

$$
\begin{aligned}
& C(15)-C(14)-C(16)-C(17) \\
& C(10)-C(14)-C(16)-C(17) \\
& C(15)-C(14)-C(16)-C(21) \\
& C(10)-C(14)-C(16)-C(21) \\
& C(21)-C(16)-C(17)-C(18) \\
& C(14)-C(16)-C(17)-C(18) \\
& C(16)-C(17)-C(18)-C(19) \\
& C(17)-C(18)-C(19)-C(20) \\
& C(17)-C(18)-C(19)-C(22) \\
& C(18)-C(19)-C(20)-C(21) \\
& C(22)-C(19)-C(20)-C(21) \\
& C(19)-C(20)-C(21)-C(16) \\
& C(17)-C(16)-C(21)-C(20) \\
& C(14)-C(16)-C(21)-C(20) \\
& H(2)-O(2)-C(22)-O(1) \\
& H(2)-O(2)-C(22)-C(19) \\
& C(20)-C(19)-C(22)-O(1) \\
& C(18)-C(19)-C(22)-O(1) \\
& C(20)-C(19)-C(22)-O(2) \\
& C(18)-C(19)-C(22)-O(2)
\end{aligned}
$$

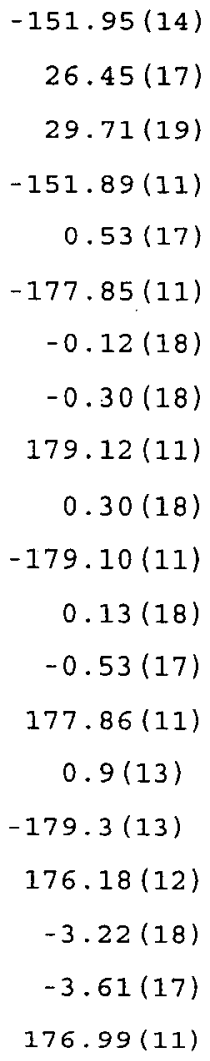


(C) 2005 American Chemical Society, Organometallics, Daiss om040143k Supporting Info Page 11 11

Table 7. Crystal data and structure refinement for $1 b$.

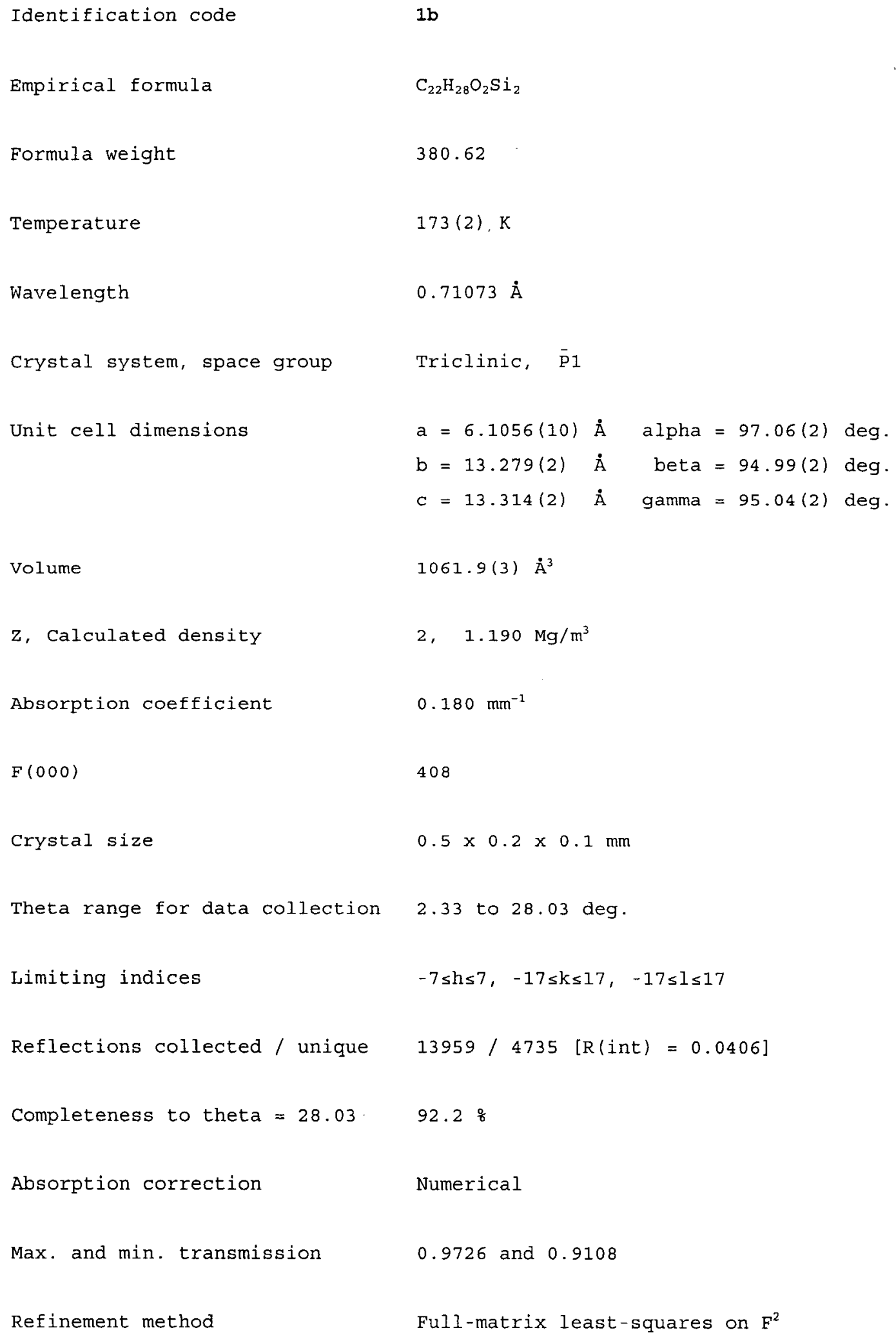


(C) 2005 American Chemical Society, Organometallics, Daiss om040143k Supporting Info Page 12

12

Data / restraints / parameters $4735 / 0 / 243$

Goodness-of-fit on $F^{2}$

1.061

Final $R$ indices [I $>2$ sigma(I)]

$R I=0.0349, w R 2=0.0949$

R indices (all data)

$R 1=0.0423, w R 2=0.0985$

Largest diff. peak and hole

0.394 and -0.272 e. $\dot{A}^{-3}$ 
(C) 2005 American Chemical Society, Organometallics, Daiss om040143k Supporting Info Page 13

13

Table 8. Atomic coordinates $\left(x 10^{4}\right)$ and equivalent isotropic displacement parameters $\left(\dot{A}^{2} \times 10^{3}\right)$ for $1 \mathrm{~b}$. $U(e q)$ is defined as one third of the trace of the orthogonalized Uij tensor.

\begin{tabular}{|c|c|c|c|c|}
\hline & $\mathrm{x}$ & $y$ & $z$ & $\mathrm{U}(\mathrm{eq})$ \\
\hline Si(1) & $11599(1)$ & $4971(1)$ & $2132(1)$ & $20(1)$ \\
\hline $\operatorname{si}(2)$ & $12501(1)$ & $3135(1)$ & $3756(1)$ & $23(1)$ \\
\hline$O(1)$ & $10583(2)$ & $194(1)$ & $-3771(1)$ & $35(1)$ \\
\hline$O(2)$ & $7715(2)$ & $739(1)$ & $-4638(1)$ & $38(1)$ \\
\hline$C(1)$ & $13442(3)$ & $5060(1)$ & $1092(1)$ & $29(1)$ \\
\hline$C(2)$ & $9570(3)$ & $5943(1)$ & $2072(1)$ & $32(1)$ \\
\hline$C(3)$ & $13316(3)$ & $5189(1)$ & $3390(1)$ & $28(1)$ \\
\hline$C(4)$ & $14510(2)$ & $4245(1)$ & $3604(1)$ & $28(1)$ \\
\hline$C(5)$ & $13980(3)$ & $1980(1)$ & $3881(2)$ & $44(1)$ \\
\hline$C(6)$ & $11007(3)$ & $3431(2)$ & $4909(1)$ & $44(1)$ \\
\hline$C(7)$ & $10407(2)$ & $2906(1)$ & $2604(1)$ & $20(1)$ \\
\hline$C(8)$ & $10073(2)$ & $3652(1)$ & $1948(1)$ & $19(1)$ \\
\hline$C(9)$ & $8520(2)$ & $3390(1)$ & $1093(1)$ & $19(1)$ \\
\hline$C(10)$ & $7251(2)$ & $2451(1)$ & $886(1)$ & $19(1)$ \\
\hline$C(11)$ & $7518(2)$ & $1725(1)$ & $1559(1)$ & $23(1)$ \\
\hline$C(12)$ & $9107(2)$ & $1968(1)$ & $2391(1)$ & $24(1)$ \\
\hline$C(13)$ & $6126(3)$ & $710(1)$ & $1394(1)$ & $34(1)$ \\
\hline$C(14)$ & $5612(2)$ & $2251(1)$ & $-43(1)$ & $20(1)$ \\
\hline$C(15)$ & $3532(2)$ & $2445(1)$ & $25(1)$ & $29(1)$ \\
\hline$C(16)$ & $6423(2)$ & $1850(1)$ & $-1021(1)$ & $20(1)$ \\
\hline$C(17)$ & $8305(2)$ & $1326(1)$ & $-1017(1)$ & $23(1)$ \\
\hline$C(18)$ & $9087(2)$ & $934(1)$ & $-1916(1)$ & $25(1)$ \\
\hline$C(19)$ & $7990(2)$ & $1056(1)$ & $-2848(1)$ & $23(1)$ \\
\hline$C(20)$ & $6106(3)$ & $1573(1)$ & $-2867(1)$ & $27(1)$ \\
\hline$C(21)$ & $5339(2)$ & $1970(1)$ & $-1965(1)$ & $25(1)$ \\
\hline$C(22)$ & $8891(3)$ & $629(1)$ & $-3792(1)$ & $26(1)$ \\
\hline
\end{tabular}


(C) 2005 American Chemical Society, Organometallics, Daiss om040143k Supporting Info Page 14

14

Table 9. Bond lengths [Aं] and angles [deg] for $1 b$.

\begin{tabular}{|c|c|}
\hline Si(1)-C(1) & $1.8665(15)$ \\
\hline $\operatorname{Si}(1)-C(2)$ & $1.8706(15)$ \\
\hline $\operatorname{Si}(1)-C(3)$ & $1.8735(14)$ \\
\hline$S i(1)-C(8)$ & $1.8877(14)$ \\
\hline$S i(2)-C(5)$ & $1.8640(17)$ \\
\hline$S i(2)-C(6)$ & $1.8711(17)$ \\
\hline $\operatorname{Si}(2)-C(4)$ & $1.8752(16)$ \\
\hline$S i(2)-C(7)$ & $1.8863(14)$ \\
\hline$O(1)-C(22)$ & $1.2269(18)$ \\
\hline$O(2)-H(2)$ & $0.83(2)$ \\
\hline$O(2)-C(22)$ & $1.3121(17)$ \\
\hline$C(3)-C(4)$ & $1.548(2)$ \\
\hline$C(7)-C(12)$ & $1.4015(19)$ \\
\hline$C(7)-C(8)$ & $1.4145(17)$ \\
\hline$C(8)-C(9)$ & $1.4047(17)$ \\
\hline$C(9)-C(10)$ & $1.3929(18)$ \\
\hline$C(10)-C(11)$ & $1.4045(17)$ \\
\hline$C(10)-C(14)$ & $1.5034(17)$ \\
\hline$C(11)-C(12)$ & $1.3932(19)$ \\
\hline$C(11)-C(13)$ & $1.5103(19)$ \\
\hline$C(14)-C(15)$ & $1.326(2)$ \\
\hline$C(14)-C(16)$ & $1.4871(17)$ \\
\hline$C(16)-C(17)$ & $1.3946(19)$ \\
\hline$C(16)-C(21)$ & $1.4022(17)$ \\
\hline$C(17)-C(18)$ & $1.3856(18)$ \\
\hline$C(18)-C(19)$ & $1.3934(18)$ \\
\hline$C(19)-C(20)$ & $1.390(2)$ \\
\hline$C(19)-C(22)$ & $1.4845(18)$ \\
\hline$C(20)-C(21)$ & $1.3873(19)$ \\
\hline$C(1)-S i(1)-C(2)$ & $109.49(7)$ \\
\hline$C(1)-S i(1)-C(3)$ & $109.32(7)$ \\
\hline$C(2)-S i(1)-C(3)$ & $110.39(7)$ \\
\hline$C(1)-S i(1)-C(8)$ & $108.49(6)$ \\
\hline$C(2)-S i(1)-C(8)$ & $109.59(7)$ \\
\hline$C(3)-S i(1)-C(8)$ & $109.53(6)$ \\
\hline$C(5)-S i(2)-C(6)$ & $109.30(9)$ \\
\hline$C(5)-S i(2)-C(4)$ & $110.23(8)$ \\
\hline$C(6)-S i(2)-C(4)$ & $110.38(8)$ \\
\hline$C(5)-S i(2)-C(7)$ & $110.58(7)$ \\
\hline
\end{tabular}




$\begin{array}{ll}C(6)-S i(2)-C(7) & 108.28(8) \\ C(4)-S i(2)-C(7) & 108.04(6) \\ H(2)-O(2)-C(22) & 106.4(15) \\ C(4)-C(3)-S i(1) & 112.09(9) \\ C(3)-C(4)-S i(2) & 111.65(10) \\ C(12)-C(7)-C(8) & 118.40(11) \\ C(12)-C(7)-S i(2) & 119.06(9) \\ C(8)-C(7)-S i(2) & 122.54(10) \\ C(9)-C(8)-C(7) & 117.85(11) \\ C(9)-C(8)-S i(1) & 117.62(9) \\ C(7)-C(8)-S i(1) & 124.53(9) \\ C(10)-C(9)-C(8) & 123.10(11) \\ C(9)-C(10)-C(11) & 119.04(11) \\ C(9)-C(10)-C(14) & 118.92(11) \\ C(11)-C(10)-C(14) & 122.03(11) \\ C(12)-C(11)-C(10) & 118.12(12) \\ C(12)-C(11)-C(13) & 120.61(12) \\ C(10)-C(11)-C(13) & 121.27(12) \\ C(11)-C(12)-C(7) & 123.41(12) \\ C(15)-C(14)-C(16) & 122.34(12) \\ C(15)-C(14)-C(10) & 120.00(11) \\ C(16)-C(14)-C(10) & 117.66(11) \\ C(17)-C(16)-C(21) & 117.92(12) \\ C(17)-C(16)-C(14) & 119.97(11) \\ C(21)-C(16)-C(14) & 122.10(12) \\ C(18)-C(17)-C(16) & 121.30(12) \\ C(17)-C(18)-C(19) & 120.15(13) \\ C(20)-C(19)-C(18) & 119.36(12) \\ C(20)-C(19)-C(22) & 122.25(12) \\ C(18)-C(19)-C(22) & 118.39(13) \\ C(21)-C(20)-C(19) & 120.22(12) \\ C(20)-C(21)-C(16) & 121.04(13) \\ O(1)-C(22)-O(2) & 123.35(12) \\ O(1)-C(22)-C(19) & 121.95(12) \\ O(2)-C(22)-C(19) & 114.69(13)\end{array}$


(C) 2005 American Chemical Society, Organometallics, Daiss om040143k Supporting Info Page 16 16

Table 10. Anisotropic displacement parameters $\left(\dot{A}^{2} \times 10^{3}\right)$ for $1 \mathrm{~b}$. The anisotropic displacement factor exponent takes the form:

$-2 \pi^{2}\left[h^{2} a \star^{2} 011+\ldots+2 h k a * b * 012\right]$

\begin{tabular}{|c|c|c|c|c|c|c|}
\hline & U11 & U2 2 & U33 & $\mathrm{U} 23$ & U13 & U12 \\
\hline Si(1) & $18(1)$ & $21(1)$ & $18(1)$ & $0(1)$ & $-1(1)$ & $-1(1)$ \\
\hline Si (2) & $22(1)$ & $29(1)$ & $16(1)$ & $2(1)$ & $-2(1)$ & $2(1)$ \\
\hline$O(1)$ & $39(1)$ & $47(1)$ & $20(1)$ & $1(1)$ & $4(1)$ & $15(1)$ \\
\hline$O(2)$ & $48(1)$ & $51(1)$ & $17(1)$ & $O(1)$ & $2(1)$ & 21 (1) \\
\hline$C(1)$ & $31(1)$ & $29(1)$ & $28(1)$ & $5(1)$ & $7(1)$ & $-2(1)$ \\
\hline$C(2)$ & $28(1)$ & $26(1)$ & $38(1)$ & $-1(1)$ & $-3(1)$ & $3(1)$ \\
\hline$C(3)$ & $30(1)$ & $28(1)$ & $23(1)$ & $-2(1)$ & $-5(1)$ & $-3(1)$ \\
\hline$C(4)$ & $22(1)$ & $36(1)$ & $23(1)$ & $3(1)$ & $-6(1)$ & $-1(1)$ \\
\hline$C(5)$ & $40(1)$ & $41(1)$ & $51(1)$ & $12(1)$ & $-10(1)$ & $10(1)$ \\
\hline$C(6)$ & $41(1)$ & $65(1)$ & $22(1)$ & $-1(1)$ & $7(1)$ & $-4(1)$ \\
\hline$C(7)$ & $21(1)$ & $24(1)$ & $15(1)$ & $1(1)$ & $1(1)$ & $2(1)$ \\
\hline$C(8)$ & $17(1)$ & $22(1)$ & $15(1)$ & $O(1)$ & $1(1)$ & $0(1)$ \\
\hline$C(9)$ & $19(1)$ & $21(1)$ & $17(1)$ & $2(1)$ & $1(1)$ & $1(1)$ \\
\hline$C(10)$ & $17(1)$ & $23(1)$ & $17(1)$ & $-2(1)$ & $2(1)$ & $2(1)$ \\
\hline$C(11)$ & $25(1)$ & $22(1)$ & $22(1)$ & $1(1)$ & $1(1)$ & $-2(1)$ \\
\hline$C(12)$ & $29(1)$ & $24(1)$ & $20(1)$ & $5(1)$ & $-1(1)$ & $0(1)$ \\
\hline$C(13)$ & $39(1)$ & $26(1)$ & $34(1)$ & $5(1)$ & $-6(1)$ & $-9(1)$ \\
\hline$C(14)$ & $20(1)$ & $19(1)$ & $19(1)$ & $-1(1)$ & $0(1)$ & $-2(1)$ \\
\hline$C(15)$ & $22(1)$ & $38(1)$ & $25(1)$ & $-5(1)$ & $0(1)$ & $2(1)$ \\
\hline$C(16)$ & $19(1)$ & $20(1)$ & $18(1)$ & $-2(1)$ & $0(1)$ & $-3(1)$ \\
\hline$C(17)$ & $22(1)$ & $29(1)$ & $17(1)$ & $1(1)$ & $-1(1)$ & $2(1)$ \\
\hline$C(18)$ & $24(1)$ & $30(1)$ & $22(1)$ & $0(1)$ & $1(1)$ & $5(1)$ \\
\hline$C(19)$ & $28(1)$ & $23(1)$ & $18(1)$ & $O(1)$ & $2(1)$ & $1(1)$ \\
\hline$C(20)$ & $32(1)$ & $30(1)$ & $18(1)$ & $2(1)$ & $-2(1)$ & $5(1)$ \\
\hline$C(21)$ & $24(1)$ & $27(1)$ & $22(1)$ & $I(1)$ & $-2(1)$ & $5(1)$ \\
\hline$C(22)$ & $34(1)$ & $25(1)$ & $19(1)$ & $1(1)$ & $3(1)$ & $3(1)$ \\
\hline
\end{tabular}


(C) 2005 American Chemical Society, Organometallics, Daiss om040143k Supporting Info Page 17 17

Table 11. Hydrogen coordinates $\left(\times 10^{4}\right)$ and isotropic displacement parameters $\left(\dot{A}^{2}\right.$ $\times 10^{3}$ ) for $1 \mathrm{~b}$.

\begin{tabular}{|c|c|c|c|c|}
\hline & $x$ & $Y$ & $z$ & $\mathrm{U}(\mathrm{eq})$ \\
\hline $\mathrm{H}(2)$ & $8330(40)$ & $452(16)$ & $-5108(17)$ & 46 \\
\hline $\mathrm{H}(1 \mathrm{~A})$ & 14672 & 4646 & 1200 & 44 \\
\hline $\mathrm{H}(1 \mathrm{~B})$ & 14021 & 5773 & 1096 & 44 \\
\hline $\mathrm{H}(1 \mathrm{C})$ & 12599 & 4808 & 436 & 44 \\
\hline $\mathrm{H}(2 \mathrm{~A})$ & 10359 & 6629 & 2209 & 47 \\
\hline $\mathrm{H}(2 \mathrm{~B})$ & 8528 & 5860 & 2583 & 47 \\
\hline $\mathrm{H}(2 \mathrm{C})$ & 8759 & 5851 & 1394 & 47 \\
\hline$H(3 A)$ & 14429 & 5780 & 3400 & 34 \\
\hline$H(3 B)$ & 12355 & 5355 & 3936 & 34 \\
\hline $\mathrm{H}(4 \mathrm{~A})$ & 15534 & 4421 & 4232 & 34 \\
\hline$H(4 B)$ & 15395 & 4050 & 3035 & 34 \\
\hline$H(5 A)$ & 15143 & 2130 & 4449 & 66 \\
\hline$H(5 B)$ & 14643 & 1783 & 3251 & 66 \\
\hline $\mathrm{H}(5 \mathrm{C})$ & 12931 & 1419 & 4009 & 66 \\
\hline $\mathrm{H}(6 \mathrm{~A})$ & 12075 & 3574 & 5514 & 66 \\
\hline $\mathrm{H}(6 \mathrm{~B})$ & 9962 & 2846 & 4978 & 66 \\
\hline $\mathrm{H}(6 \mathrm{C})$ & 10200 & 4029 & 4838 & 66 \\
\hline $\mathrm{H}(9)$ & 8326 & 3876 & 634 & 23 \\
\hline $\mathrm{H}(12)$ & 9320 & 1471 & 2837 & 29 \\
\hline$H(13 A)$ & 4561 & 822 & 1295 & 51 \\
\hline $\mathrm{H}(13 \mathrm{~B})$ & 6382 & 354 & 1989 & 51 \\
\hline$H(13 C)$ & 6531 & 296 & 789 & 51 \\
\hline $\mathrm{H}(15 \mathrm{~A})$ & 2501 & 2328 & -564 & 35 \\
\hline $\mathrm{H}(15 \mathrm{~B})$ & 3073 & 2701 & 663 & 35 \\
\hline $\mathrm{H}(17)$ & 9066 & 1236 & -386 & 28 \\
\hline $\mathrm{H}(18)$ & 10375 & 581 & -1896 & 30 \\
\hline $\mathrm{H}(20)$ & 5341 & 1655 & -3500 & 32 \\
\hline $\mathrm{H}(21)$ & 4059 & 2328 & -1987 & 30 \\
\hline
\end{tabular}


Table 12. Torsion angles [deg] for $1 b$.

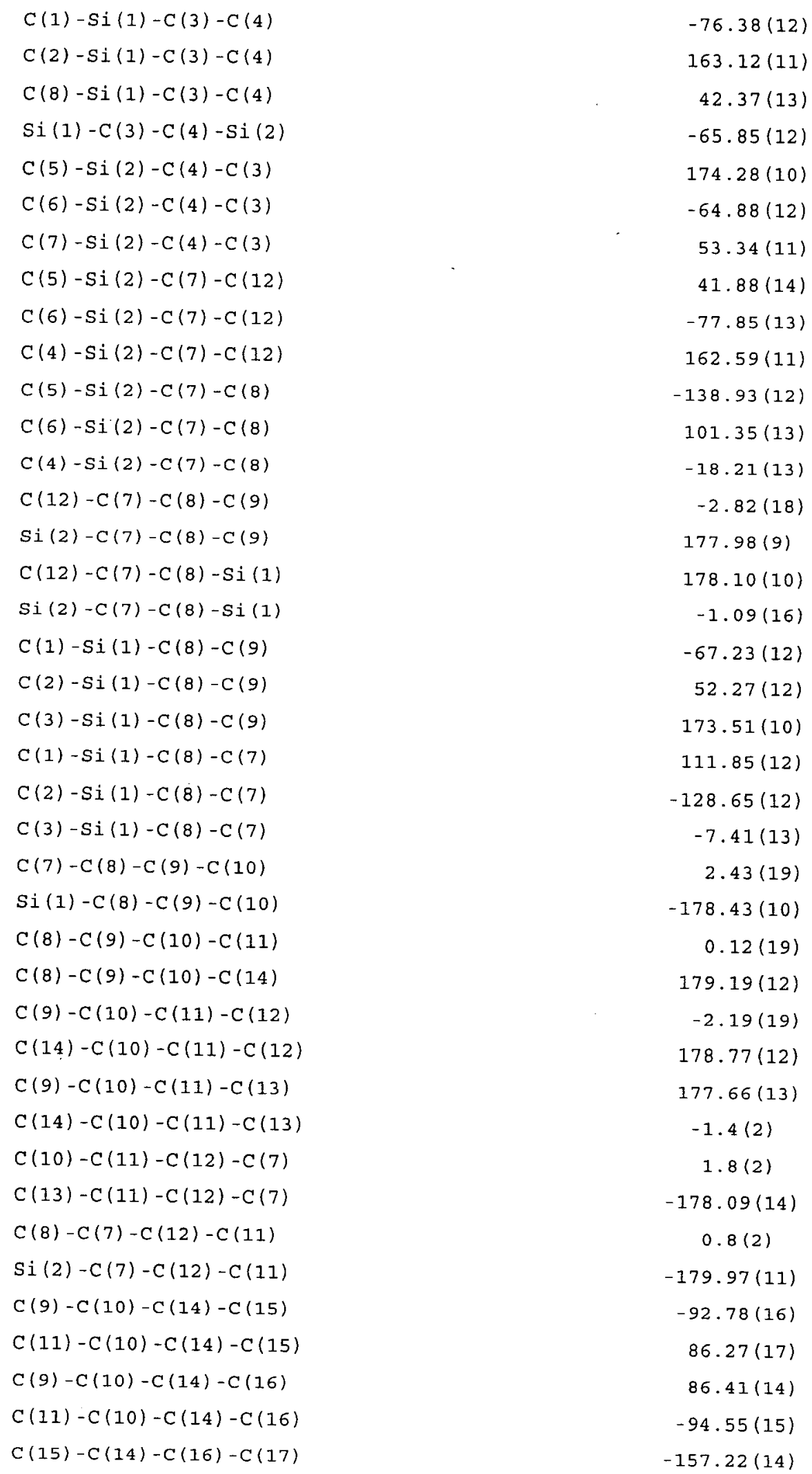

$-76.38(12)$

$163.12(11)$

$42.37(13)$

$-65.85(12)$

$174.28(10)$

$-64.88(12)$

$53.34(11)$

$41.88(14)$

$-77.85(13)$

$162.59(11)$

$-138.93(12)$

$101.35(13)$

-18.21 (13)

$-2.82(18)$

$177.98(9)$

$178.10(10)$

$-1.09(16)$

$-67.23(12)$

$52.27(12)$

$173.51(10)$

$111.85(12)$

$-128.65(12)$

$-7.41(13)$

2.43 (19)

$-178.43(10)$

$0.12(19)$

$179.19(12)$

$-2.19(19)$

$178.77(12)$

$177.66(13)$

$-1.4(2)$

$1.8(2)$

$-178.09(14)$

$0.8(2)$

$-179.97(11)$

$-92.78(16)$

$86.27(17)$

86.41 (14)

$-94.55(15)$

-157.22 (14) 
(C) 2005 American Chemical Society, Organometallics, Daiss om040143k Supporting Info Page 19 19

$$
\begin{aligned}
& C(10)-C(14)-C(16)-C(17) \\
& C(15)-C(14)-C(16)-C(21) \\
& C(10)-C(14)-C(16)-C(21) \\
& C(21)-C(16)-C(17)-C(18) \\
& C(14)-C(16)-C(17)-C(18) \\
& C(16)-C(17)-C(18)-C(19) \\
& C(17)-C(18)-C(19)-C(20) \\
& C(17)-C(18)-C(19)-C(22) \\
& C(18)-C(19)-C(20)-C(21) \\
& C(22)-C(19)-C(20)-C(21) \\
& C(19)-C(20)-C(21)-C(16) \\
& C(17)-C(16)-C(21)-C(20) \\
& C(14)-C(16)-C(21)-C(20) \\
& H(2)-O(2)-C(22)-O(1) \\
& H(2)-O(2)-C(22)-C(19) \\
& C(20)-C(19)-C(22)-O(1) \\
& C(18)-C(19)-C(22)-O(1) \\
& C(20)-C(19)-C(22)-O(2) \\
& C(18)-C(19)-C(22)-O(2)
\end{aligned}
$$

$$
\begin{gathered}
23.62(17) \\
22.1(2) \\
-157.07(12) \\
0.0(2) \\
179.33(12) \\
-0.2(2) \\
-0.1(2) \\
179.68(13) \\
0.5(2) \\
-179.24(13) \\
-0.7(2) \\
0.4(2) \\
-178.88(13) \\
1.4(16) \\
-177.3(16) \\
178.34(14) \\
-1.4(2) \\
-2.9(2) \\
177.29(13)
\end{gathered}
$$


(C) 2005 American Chemical Society, Organometallics, Daiss om040143k Supporting Info Page 20

20

Table 13. Crystal data and structure refinement for 8 .

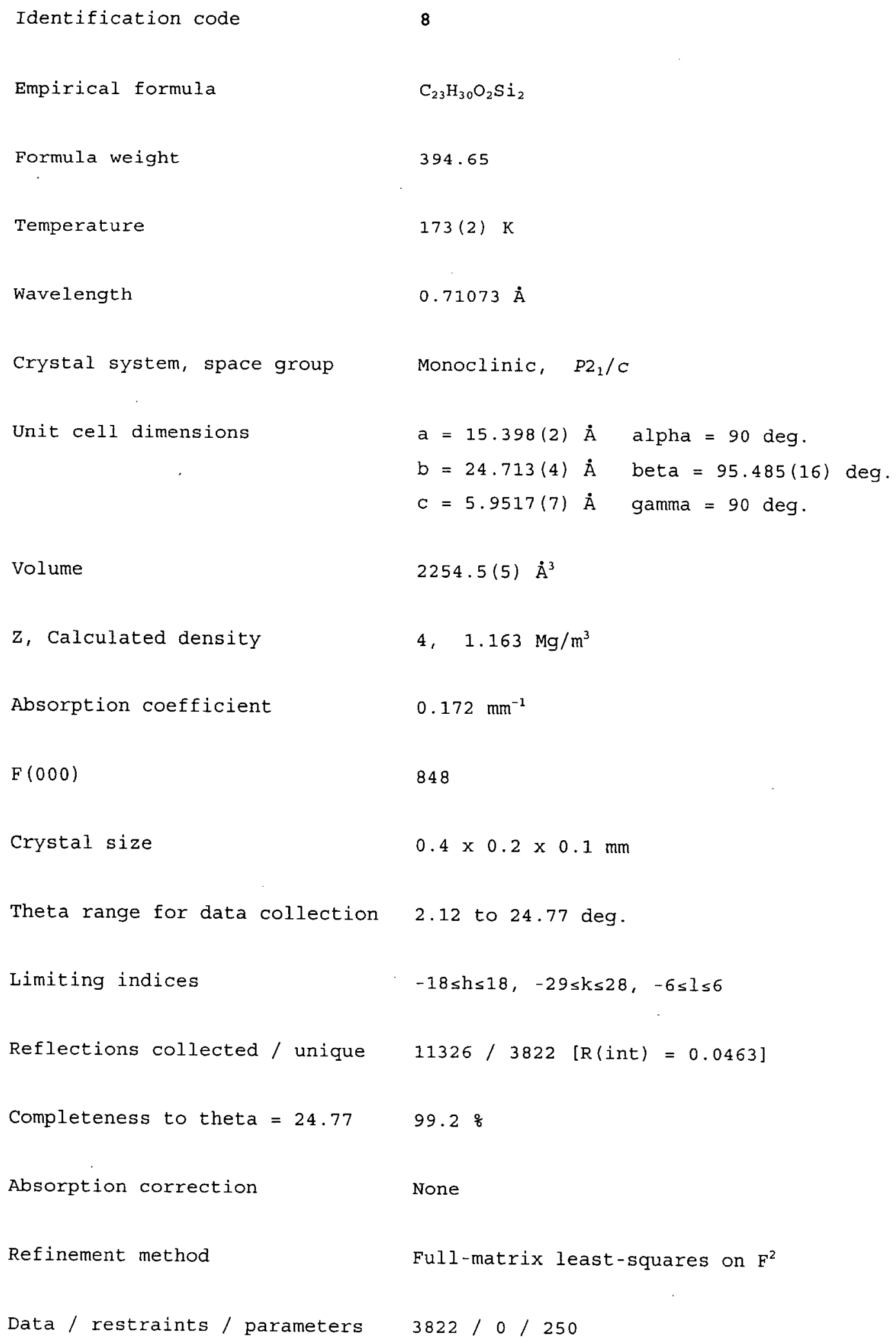


(C) 2005 American Chemical Society, Organometallics, Daiss om040143k Supporting Info Page 21 21

$\begin{array}{ll}\text { Goodness-of-fit on } F^{2} & 0.915 \\ \text { Final R indices [I>2sigma(I)] } & R 1=0.0342, \text { WR2 }=0.0777 \\ \text { R indices (all data) } & R 1=0.0554, \text { WR2 }=0.0831 \\ \text { Largest diff. peak and hole } & 0.221 \text { and }-0.143 \mathrm{e} \cdot \dot{A}^{-3}\end{array}$


(C) 2005 American Chemical Society, Organometallics, Daiss om040143k Supporting Info Page 22

22

Table 14. Atomic coordinates $\left(x 10^{4}\right)$ and equivalent isotropic displacement parameters $\left(\dot{A}^{2} \times 10^{3}\right)$ for 8 . $U(e q)$ is defined as one third of the trace of the orthogonalized Uij tensor.

\begin{tabular}{|c|c|c|c|c|}
\hline & $\mathrm{x}$ & $y$ & $z$ & $\mathrm{U}(\mathrm{eq})$ \\
\hline $\operatorname{si}(1)$ & $3487(1)$ & $3699(1)$ & $2952(1)$ & $28(1)$ \\
\hline $\operatorname{Si}(2)$ & $3050(1)$ & $5063(1)$ & $3766(1)$ & $28(1)$ \\
\hline$O(1)$ & $-3046(1)$ & $3296(1)$ & $726(2)$ & $35(1)$ \\
\hline$O(2)$ & $-3558(1)$ & $3041(1)$ & $3972(3)$ & $40(1)$ \\
\hline$C(1)$ & $4367(1)$ & $3544(1)$ & $5222(4)$ & $43(1)$ \\
\hline$C(2)$ & $3261(1)$ & $3079(1)$ & $1195(4)$ & $45(1)$ \\
\hline$C(3)$ & $3825(1)$ & $4258(1)$ & $1101(4)$ & $37(1)$ \\
\hline$C(4)$ & $4015(1)$ & $4786(1)$ & $2444(4)$ & $37(1)$ \\
\hline$C(5)$ & $3460(1)$ & $5486(1)$ & $6236(4)$ & $40(1)$ \\
\hline$C(6)$ & $2333(1)$ & $5482(1)$ & $1749(4)$ & $47(1)$ \\
\hline$C(7)$ & $2360(1)$ & $4493(1)$ & $4729(3)$ & $27(1)$ \\
\hline$C(8)$ & $2501(1)$ & $3938(1)$ & $4334(3)$ & $25(1)$ \\
\hline$C(9)$ & $1906(1)$ & $3567(1)$ & $5101(3)$ & $28(1)$ \\
\hline$C(10)$ & $1193(1)$ & $3724(1)$ & $6235(3)$ & $29(1)$ \\
\hline$C(11)$ & $1063(1)$ & $4273(1)$ & $6654(4)$ & $34(1)$ \\
\hline$C(12)$ & $1644(1)$ & $4642(1)$ & $5881(4)$ & $33(1)$ \\
\hline$C(13)$ & $333(2)$ & $4470(1)$ & $7975(5)$ & $54(1)$ \\
\hline$C(14)$ & $593(1)$ & $3300(1)$ & $7011(3)$ & $31(1)$ \\
\hline$C(15)$ & $883(1)$ & $2932(1)$ & $8504(5)$ & $53(1)$ \\
\hline$C(16)$ & $-323(1)$ & $3286(1)$ & $5959(3)$ & $26(1)$ \\
\hline$C(17)$ & $-987(1)$ & $3058(1)$ & $7104(3)$ & $29(1)$ \\
\hline$C(18)$ & $-1835(1)$ & $3035(1)$ & $6098(3)$ & $28(1)$ \\
\hline$C(19)$ & $-2041(1)$ & $3229(1)$ & $3943(3)$ & $25(1)$ \\
\hline$C(20)$ & $-1389(1)$ & $3466(1)$ & $2791(3)$ & $29(1)$ \\
\hline$C(21)$ & $-544(1)$ & $3495(1)$ & $3811(3)$ & $30(1)$ \\
\hline$C(22)$ & $-2960(1)$ & $3179(1)$ & $2920(3)$ & $28(1)$ \\
\hline$C(23)$ & $-3925(1)$ & $3269(1)$ & $-370(4)$ & $44(1)$ \\
\hline
\end{tabular}


(C) 2005 American Chemical Society, Organometallics, Daiss om040143k Supporting Info Page 23

23

Table 15. Bond lengths [Aं] and angles [deg] for 8 .

\begin{tabular}{|c|c|}
\hline$S i(1)-C(1)$ & $1.859(2)$ \\
\hline$S i(1)-C(2)$ & $1.869(2)$ \\
\hline$S i(1)-C(3)$ & $1.873(2)$ \\
\hline $\mathrm{Si}(1)-\mathrm{C}(8)$ & $1.8892(18)$ \\
\hline Si (2) $-C(5)$ & $1.864(2)$ \\
\hline $\operatorname{Si}(2)-C(6)$ & $1.865(2)$ \\
\hline$S i(2)-C(4)$ & $1.875(2)$ \\
\hline $\operatorname{Si}(2)-C(7)$ & $1.8874(19)$ \\
\hline$O(1)-C(22)$ & $1.332(2)$ \\
\hline$O(1)-C(23)$ & $1.447(2)$ \\
\hline$O(2)-C(22)$ & $1.209(2)$ \\
\hline$C(3)-C(4)$ & $1.544(3)$ \\
\hline$C(7)-C(12)$ & $1.403(3)$ \\
\hline$C(7)-C(8)$ & $1.411(3)$ \\
\hline$C(8)-C(9)$ & $1.402(3)$ \\
\hline$C(9)-C(10)$ & $1.397(3)$ \\
\hline$c(10)-c(11)$ & $1.396(3)$ \\
\hline$C(10)-C(14)$ & $1.499(3)$ \\
\hline$C(11)-C(12)$ & $1.387(3)$ \\
\hline$C(11)-C(13)$ & $1.513(3)$ \\
\hline$C(14)-C(15)$ & $1.320(3)$ \\
\hline$C(14)-C(16)$ & $1.489(2)$ \\
\hline$C(16)-C(21)$ & $1.390(3)$ \\
\hline$C(16)-C(17)$ & $1.400(3)$ \\
\hline$C(17)-C(18)$ & $1.385(3)$ \\
\hline$C(18)-C(19)$ & $1.378(3)$ \\
\hline$C(19)-C(20)$ & $1.397(3)$ \\
\hline$C(19)-C(22)$ & $1.492(2)$ \\
\hline$C(20)-C(21)$ & $1.384(3)$ \\
\hline$C(1)-S i(1)-C(2)$ & $108.77(11)$ \\
\hline$C(1)-\operatorname{Si}(1)-C(3)$ & $110.82(10)$ \\
\hline$C(2)-S i(1)-C(3)$ & $108.86(11)$ \\
\hline$C(1)-\operatorname{Si}(1)-C(8)$ & $107.96(9)$ \\
\hline$C(2)-S i(1)-C(8)$ & $112.67(9)$ \\
\hline$C(3)-S i(1)-C(8)$ & $107.77(9)$ \\
\hline$C(5)-S i(2)-C(6)$ & $109.38(11)$ \\
\hline$C(5)-S i(2)-C(4)$ & $108.27(9)$ \\
\hline$C(6)-S i(2)-C(4)$ & $112.11(11)$ \\
\hline$C(5)-S i(2)-C(7)$ & $109.70(9)$ \\
\hline
\end{tabular}


(C) 2005 American Chemical Society, Organometallics, Daiss om040143k Supporting Info Page 24 24

\begin{tabular}{ll}
$C(6)-S i(2)-C(7)$ & $107.07(9)$ \\
$C(4)-S i(2)-C(7)$ & $110.28(9)$ \\
$C(22)-O(1)-C(23)$ & $115.61(16)$ \\
$C(4)-C(3)-S i(1)$ & $111.66(15)$ \\
$C(3)-C(4)-S i(2)$ & $114.03(13)$ \\
$C(12)-C(7)-C(8)$ & $118.54(16)$ \\
$C(12)-C(7)-S i(2)$ & $116.33(14)$ \\
$C(8)-C(1)-S i(2)$ & $125.11(13)$ \\
$C(9)-C(8)-C(7)$ & $117.69(16)$ \\
$C(9)-C(8)-S i(1)$ & $120.97(14)$ \\
$C(7)-C(8)-S i(1)$ & $121.27(13)$ \\
$C(10)-C(9)-C(8)$ & $122.93(17)$ \\
$C(11)-C(10)-C(9)$ & $119.26(17)$ \\
$C(11)-C(10)-C(14)$ & $121.38(16)$ \\
$C(9)-C(10)-C(14)$ & $119.35(17)$ \\
$C(12)-C(11)-C(10)$ & $118.14(17)$ \\
$C(12)-C(11)-C(13)$ & $119.70(18)$ \\
$C(10)-C(11)-C(13)$ & $122.13(18)$ \\
$C(11)-C(12)-C(7)$ & $123.43(18)$ \\
$C(15)-C(14)-C(16)$ & $120.82(17)$ \\
$C(15)-C(14)-C(10)$ & $120.79(17)$ \\
$C(16)-C(14)-C(10)$ & $118.30(16)$ \\
$C(21)-C(16)-C(17)$ & $118.15(16)$ \\
$C(21)-C(16)-C(14)$ & $121.09(16)$ \\
$C(17)-C(16)-C(14)$ & $120.75(17)$ \\
$C(18)-C(17)-C(16)$ & $120.53(18)$ \\
$C(19)-C(18)-C(17)$ & $120.77(17)$ \\
$C(18)-C(19)-C(20)$ & $119.37(16)$ \\
$C(18)-C(19)-C(22)$ & $118.38(16)$ \\
$C(20)-C(19)-C(22)$ & $122.25(18)$ \\
$C(21)-C(20)-C(19)$ & $119.75(18)$ \\
$C(20)-C(21)-C(16)$ & $121.39(17)$ \\
$O(2)-C(22)-O(1)$ & $124.03(17)$ \\
$O(2)-C(22)-C(19)$ & $123.48(19)$ \\
$O(1)-C(22)-C(19)$ & $112.49(16)$ \\
& \\
\hline &
\end{tabular}


(C) 2005 American Chemical Society, Organometallics, Daiss om040143k Supporting Info Page 25

25

Table 16. Anisotropic displacement parameters $\left(\dot{A}^{2} \times 10^{3}\right)$ for 8 . The anisotropic displacement factor exponent takes the form:

$-2 \pi^{2}\left[h^{2} a \star^{2} 011+\ldots+2 h k a * b * U 12\right]$

\begin{tabular}{|c|c|c|c|c|c|c|}
\hline & U11 & U22 & U33 & U2 3 & $\mathrm{U} 13$ & U12 \\
\hline $\operatorname{si}(1)$ & $23(1)$ & $32(1)$ & $29(1)$ & $-4(1)$ & $3(1)$ & $2(1)$ \\
\hline Si (2) & $26(1)$ & $28(1)$ & $32(1)$ & $1(1)$ & $7(1)$ & $0(1)$ \\
\hline$O(1)$ & $27(1)$ & $46(1)$ & $32(1)$ & $0(1)$ & $-1(1)$ & $4(1)$ \\
\hline$O(2)$ & $25(1)$ & $51(1)$ & $44(1)$ & $4(1)$ & $6(1)$ & $-4(1)$ \\
\hline$C(1)$ & $28(1)$ & $60(2)$ & $39(1)$ & $0(1)$ & $2(1)$ & $10(1)$ \\
\hline$C(2)$ & $41(1)$ & $45(1)$ & $49(2)$ & $-13(1)$ & $8(1)$ & $1(1)$ \\
\hline$C(3)$ & $33(1)$ & $45(1)$ & $33(1)$ & $-4(1)$ & $10(1)$ & $1(1)$ \\
\hline$C(4)$ & $29(1)$ & $40(1)$ & $43(1)$ & $-1(1)$ & $11(1)$ & $-4(1)$ \\
\hline$C(5)$ & $39(1)$ & $35(1)$ & $47(2)$ & $-6(1)$ & $10(1)$ & $-6(1)$ \\
\hline$C(6)$ & $39(1)$ & $49(1)$ & $52(2)$ & $15(1)$ & $5(1)$ & $2(1)$ \\
\hline$C(7)$ & $23(1)$ & $31(1)$ & $27(1)$ & $0(1)$ & $1(1)$ & $0(1)$ \\
\hline$C(8)$ & $22(1)$ & $31(1)$ & $23(1)$ & $-1(1)$ & $-1(1)$ & $-1(1)$ \\
\hline$C(9)$ & $28(1)$ & $26(1)$ & $30(1)$ & $-1(1)$ & $-1(1)$ & $2(1)$ \\
\hline$C(10)$ & $24(1)$ & $33(1)$ & $28(1)$ & $1(1)$ & $1(1)$ & $-4(1)$ \\
\hline$C(11)$ & $27(1)$ & $38(1)$ & $37(1)$ & $-5(1)$ & $9(1)$ & $-1(1)$ \\
\hline$C(12)$ & $31(1)$ & $26(1)$ & $43(1)$ & $-7(1)$ & $7(1)$ & $1(1)$ \\
\hline$C(13)$ & $47(1)$ & $45(1)$ & $77(2)$ & $-15(1)$ & $32(1)$ & $-5(1)$ \\
\hline$C(14)$ & $27(1)$ & $34(1)$ & $33(1)$ & $2(1)$ & $4(1)$ & $-2(1)$ \\
\hline$C(15)$ & $28(1)$ & $61(2)$ & $69(2)$ & $28(1)$ & $-7(1)$ & $-9(1)$ \\
\hline$C(16)$ & $26(1)$ & $25(1)$ & $29(1)$ & $-1(1)$ & $5(1)$ & $0(1)$ \\
\hline$C(17)$ & $31(1)$ & $31(1)$ & $25(1)$ & $5(1)$ & $5(1)$ & $-1(1)$ \\
\hline$C(18)$ & $26(1)$ & $29(1)$ & $31(1)$ & $4(1)$ & $9(1)$ & $-1(1)$ \\
\hline$C(19)$ & $25(1)$ & $23(1)$ & $29(1)$ & $-2(1)$ & $5(1)$ & $2(1)$ \\
\hline$C(20)$ & $29(1)$ & $34(1)$ & $25(1)$ & $6(1)$ & $4(1)$ & $2(I)$ \\
\hline$C(21)$ & $26(1)$ & $34(1)$ & $33(1)$ & $7(1)$ & $7(1)$ & $-4(1)$ \\
\hline$C(22)$ & $27(1)$ & $22(1)$ & $35(1)$ & $-3(1)$ & $4(1)$ & $3(1)$ \\
\hline$C(23)$ & $31(1)$ & $59(1)$ & $41(1)$ & $-5(1)$ & $-9(1)$ & $6(1)$ \\
\hline
\end{tabular}


(C) 2005 American Chemical Society, Organometallics, Daiss om040143k Supporting Info Page 26 26

- Table 17. Hydrogen coordinates $\left(\times 10^{4}\right)$ and isotropic displacement parameters $\left(\dot{A}^{2}\right.$ $x 10^{3}$ ) for 8 .

\begin{tabular}{|c|c|c|c|c|}
\hline & $x$ & $y$ & $z$ & $\mathrm{U}(\mathrm{eq})$ \\
\hline$H(I A)$ & 4891 & 3427 & 4544 & 51 \\
\hline $\mathrm{H}(1 \mathrm{~B})$ & 4173 & 3254 & 6183 & 51 \\
\hline $\mathrm{H}(\mathrm{IC})$ & 4499 & 3868 & 6136 & 51 \\
\hline $\mathrm{H}(2 \mathrm{~A})$ & 3810 & 2933 & 747 & 53 \\
\hline $\mathrm{H}(2 \mathrm{~B})$ & 2876 & 3172 & -157 & 53 \\
\hline $\mathrm{H}(2 \mathrm{C})$ & 2977 & 2806 & 2073 & 53 \\
\hline$H(3 A)$ & 4354 & 4147 & 398 & 44 \\
\hline$H(3 B)$ & 3355 & 4324 & -123 & 44 \\
\hline$H(4 A)$ & 4222 & 5063 & 1418 & 44 \\
\hline$H(4 B)$ & 4492 & 4718 & 3647 & 44 \\
\hline$H(5 A)$ & 3812 & 5784 & 5727 & 48 \\
\hline$H(5 B)$ & 3819 & 5263 & 7324 & 48 \\
\hline $\mathrm{H}(5 \mathrm{C})$ & 2964 & 5633 & 6956 & 48 \\
\hline $\mathrm{H}(6 \mathrm{~A})$ & 2664 & 5791 & 1254 & 56 \\
\hline $\mathrm{H}(6 \mathrm{~B})$ & 1833 & 5612 & 2495 & 56 \\
\hline $\mathrm{H}(6 \mathrm{C})$ & 2126 & 5261 & 436 & 56 \\
\hline $\mathrm{H}(9)$ & 1991 & 3193 & 4839 & 34 \\
\hline $\mathrm{H}(12)$ & 1552 & 5016 & 6145 & 39 \\
\hline$H(13 A)$ & 384 & 4862 & 8193 & 65 \\
\hline $\mathrm{H}(13 \mathrm{~B})$ & 372 & 4290 & 9448 & 65 \\
\hline $\mathrm{H}(13 \mathrm{C})$ & -230 & 4385 & 7139 & 65 \\
\hline$H(15 A)$ & 505 & 2653 & 8919 & 64 \\
\hline$H(15 B)$ & 1469 & 2945 & 9163 & 64 \\
\hline $\mathrm{H}(17)$ & -854 & 2918 & 8583 & 35 \\
\hline$H(18)$ & -2280 & 2883 & 6902 & 34 \\
\hline$H(20)$ & -1525 & 3606 & 1313 & 35 \\
\hline $\mathrm{H}(21)$ & -105 & 3661 & 3027 & 36 \\
\hline$H(23 A)$ & -3912 & 3321 & -1999 & 53 \\
\hline $\mathrm{H}(23 \mathrm{~B})$ & -4280 & 3554 & 233 & 53 \\
\hline$H(23 C)$ & -4178 & 2915 & -85 & 53 \\
\hline
\end{tabular}


(C) 2005 American Chemical Society, Organometallics, Daiss om040143k Supporting Info Page 27

Table 18. Torsion angles [deg] for 8 .

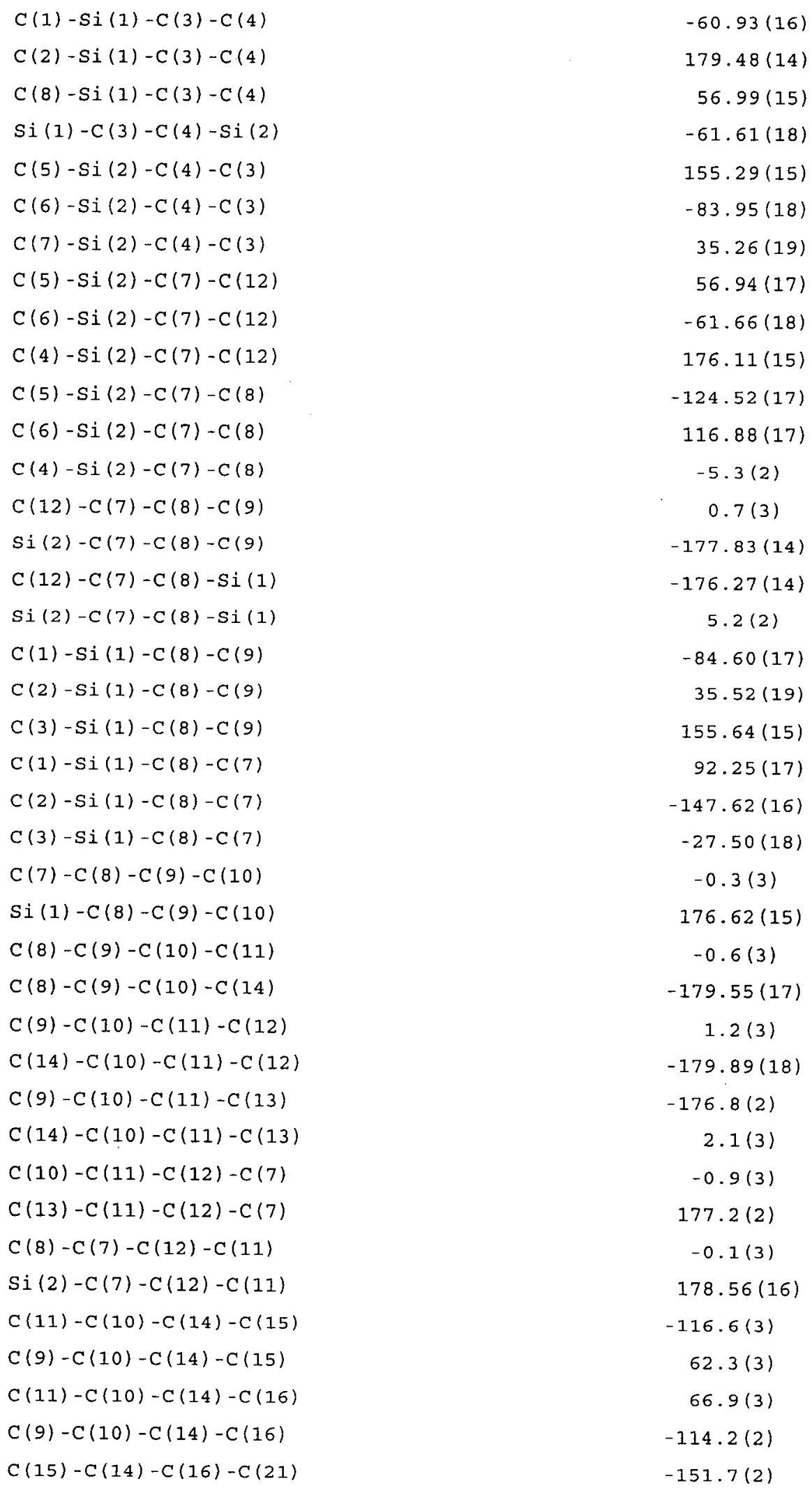


(C) 2005 American Chemical Society, Organometallics, Daiss om040143k Supporting Info Page 28

$$
\begin{aligned}
& C(10)-C(14)-C(16)-C(21) \\
& C(15)-C(14)-C(16)-C(17) \\
& C(10)-C(14)-C(16)-C(17) \\
& C(21)-C(16)-C(17)-C(18) \\
& C(14)-C(16)-C(17)-C(18) \\
& C(16)-C(17)-C(18)-C(19) \\
& C(17)-C(18)-C(19)-C(20) \\
& C(17)-C(18)-C(19)-C(22) \\
& C(18)-C(19)-C(20)-C(21) \\
& C(22)-C(19)-C(20)-C(21) \\
& C(19)-C(20)-C(21)-C(16) \\
& C(17)-C(16)-C(21)-C(20) \\
& C(14)-C(16)-C(21)-C(20) \\
& C(23)-O(1)-C(22)-O(2) \\
& C(23)-O(1)-C(22)-C(19) \\
& C(18)-C(19)-C(22)-O(2) \\
& C(20)-C(19)-C(22)-O(2) \\
& C(18)-C(19)-C(22)-O(1) \\
& C(20)-C(19)-C(22)-O(1)
\end{aligned}
$$

$$
\begin{gathered}
24.8(3) \\
27.8(3) \\
-155.64(18) \\
1.0(3) \\
-178.55(17) \\
0.8(3) \\
-1.7(3) \\
178.20(17) \\
0.9(3) \\
-179.05(17) \\
0.9(3) \\
-1.9(3) \\
177.69(18) \\
2.1(3) \\
-178.46(16) \\
10.0(3) \\
-170.07(18) \\
-169.44(16) \\
10.5(2)
\end{gathered}
$$

